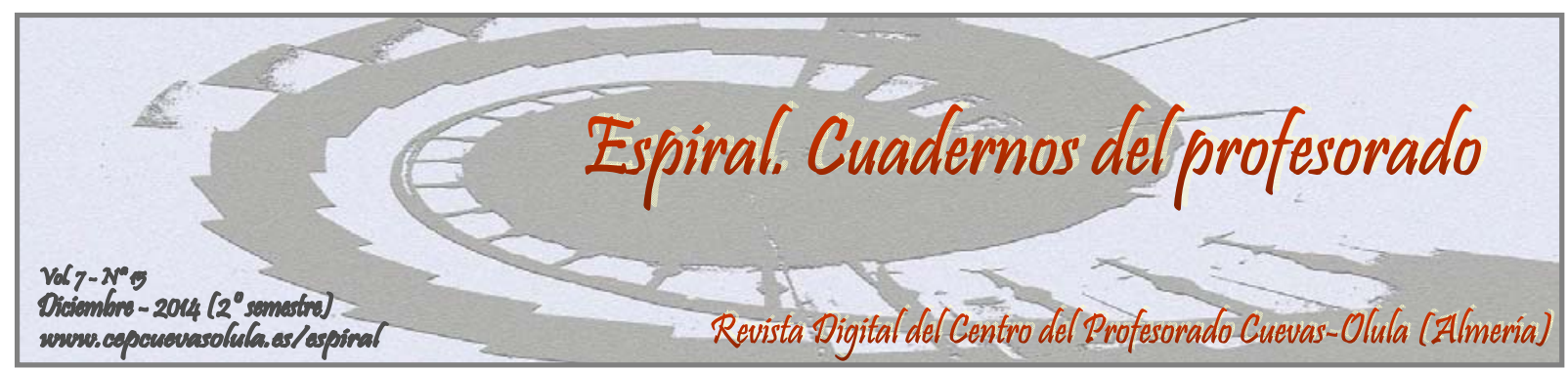

\title{
CÓMO CONSTRUIR UN ROCÓDROMO DE ESCALADA: IDEAS PARA EDUCACIÓN FÍSICA
}

\section{HOW TO BUILD A CLIMBIMG WALL: IDEAS FOR PHYSICAL EDUCATION}

\author{
Antonio Baena-Extremera ${ }^{(1)}$, José David Ayala-Jiménez ${ }^{(2)}$ \\ y José David Meroño Subira ${ }^{(3)}$
}

(1) Facultad de Ciencias del Deporte, Universidad de Murcia, España

(2) Facultad de Ciencias del Deporte, Universidad Europea de Madrid, España

(3) Facultad de Química, Universidad de Murcia, España

RESUMEN: La escalada es un recurso cada vez más utilizado en las clases de educación física, dada la importancia que le da el currículum oficial, así como lo atrayente y motivante que resulta para nuestro alumnado este tipo de actividades. Con esta propuesta se pretende dotar a los docentes de un nuevo modelo para llevar a cabo la construcción de un rocódromo en los centros educativos. En este artículo se pretende desglosar cómo construir un rocódromo paso a paso, desde la planificación hasta la construcción y utilización del mismo. Asimismo, se ejemplifica cómo se lleva a cabo la construcción de uno real.

Palabras clave: rocódromo, escalada, actividad física en el medio natural, construcción.

ABSTRACT: Climbing is becoming a resource increasingly used in Physical Education lessons, as for its importance to the official curriculum and, for the interest and motivation these activities cause in our students. This proposal tries to provide teachers with a new model to accomplish the construction of a climbing wall in Educational Centres. This article aims to explain in detail how to build a climbing wall step by step, from its planning to its construction and utilization. To the same extent, it is exemplified how to accomplish the construction of a real one.

Key words: climbing wall, climbing, physical activities in the natural environment, construction.

Baena-Extremera, A., Ayala-Jiménez, J. D., y Meroño Subira, P. J. (2014). Cómo construir un rocódromo de escalada: ideas para Educación Física. Espiral. Cuadernos del Profesorado, 7(15), 36-41. Disponible en: http://www.cepcuevasolula.es/espiral.

Fecha de recepción: 17/04/2014

Fecha de aceptación: 20/08/2014
Enviar correspondencia a: abaenaextrem@um.es

\section{1.- INTRODUCCIÓN}

La escalada es una actividad en el medio natural que se basa en la habilidad motriz de la trepa y que por el riesgo que implica requiere el empleo de determinadas técnicas de aseguramiento. (Aguado, 2001).

En Educación Física, una unidad didáctica de escalada se puede llevar a cabo en las espalderas, en un rocódromo e incluso en el medio natural. Una instalación artificial muy útil para trabajar estos contenidos es un rocódromo de escalada, que podemos construir de manera barata y 
rápida. Los beneficios que nos otorga esta utilización, son enormes, pues por un lado, no hace falta sacar a los alumnos del centro para practicar este deporte, la incertidumbre del medio se controla a priori, se conoce de antemano el recorrido y las presas donde agarrarse, etc.

La iniciativa que a continuación se explica, surge debido al auge de los contenidos de actividad física en el medio natural en Educación Física (Baena y Granero 2009; Baena y Fernández 2013), como muchos autores dejan explícito. Además, para poder trabajar estos contenidos y otros contenidos de medio natural, se están en los últimos años publicando diversos trabajos sobre la construcción de este tipo instalaciones, para ofrecerle al profesorado herramientas para afrontar las carencias de instalaciones de los centros educativos. Algunos ejemplos, se pueden ver en el trabajo de Baena-Extremera (2011), en la elaboración de un recorrido simulando un itinerario en espeleología, o la adaptación de una vía ferrata en el centro educativo (Baena-Extremera y Fernández 2013)

Esta propuesta nace también como respuesta a las inquietudes del profesorado para construir instalaciones que las consejerías de educación no satisfacen en los centros, y ante la necesidad de tener que desarrollar los contenidos del bloque medio natural (Baena y Calvo, 2008; Beas y Blanes 2010; Briongos y Pérez, 2008; Casero, 2007; Sánchez, 2009) y dentro del mismo, juegos y ejercicios de trepa y escalada, como nos deja explícito el Real decreto 1631/2006, de 29 de diciembre.

Por todo ello, a continuación se propone una forma alternativa de cómo construir un rocódromo, que permita una mayor transferencia para nuestros alumnos a la realidad. Entre las novedades de este trabajo con lo ya conocido por otros autores, es que dicha propuesta trata de un rocódromo abatible, que aporta una mayor creatividad y flexibilidad a la hora de desarrollar ejercicios prácticos sobre él.

Con este tipo de estructuras se pretende añadir un mayor realismo a la hora de comparar los ejercicios propuestos con los que encontraríamos en el medio natural, en una futura salida a una zona de escalada en roca. De esta manera buscamos una mayor implicación y motivación por parte del alumnado en las tareas propuestas.

\section{Referencia al Currículo de educación Física}

Esta propuesta de trabajo se enmarca dentro del currículo de la Enseñanza Secundaria Obligatoria (E.S.O.), R.D. 1631/2006, de 29 de diciembre, por el que se establecen las enseñanzas mínimas en E.S.O. No obstante, estos contenidos estarían también presentes en el currículum de primaria, a través de diversos bloques que trabajen los juegos y la iniciación deportiva.

La escalada nos permitirá contribuir directamente en mayor o menor grado al desarrollo de las siguientes capacidades:

- Realizar tareas dirigidas al incremento de las posibilidades de rendimiento motor, a la mejora de la condición física para la salud y al perfeccionamiento de las funciones de ajuste, dominio y control corporal, adoptando una actitud de autoexigencia en su ejecución.

- Planificar actividades que permitan satisfacer las necesidades en relación a las capacidades físicas y habilidades específicas a partir de la valoración del nivel inicial.

- Realizar actividades físico-deportivas en el medio natural que tengan bajo impacto ambiental, contribuyendo a su conservación.

- Conocer y realizar actividades deportivas y recreativas individuales, aplicando los fundamentos técnicos en situaciones reales con progresiva autonomía en su ejecución.

El principal bloque de contenidos en el que llevar a cabo este tipo de actividades en el área de Educación Física sería el de Actividades en el Medio Natural. Aunque también se puede incluir en el de Condición Física y Salud y en el de Juegos y Deportes.

En cuanto a la interdisciplinariedad para contribuir el desarrollo integral del alumno, consideramos siguiendo a Baena y Calvo (2008), que esta propuesta tiene relación con las siguientes áreas:

- Matemáticas: contenidos relacionados con trayectorias, fuerzas, presiones...

- Dibujo: líneas rectas, ángulos, geomorfología de las presas. 
- Ciencias Naturales: respeto por el medio natural y verlo como un lugar de práctica deportiva, ayuda al conocimiento del cuerpo, al conocimiento de los materiales...

- Física: de manera interdisciplinar se habla de fuerza de gravedad, magnitud de resistencia de los materiales, agarres, ángulos y tracciones, poleas...

- Lenguaje: vocabulario especifico como chapas, rocódromo, mosquetón...

- Tecnología: material y herramientas para construir el rocódromo...

\section{2.- EL ROCÓDROMO}

Siguiendo las ideas de Briongos y Pérez (2008), un rocódromo se puede definir como una estructura artificial, que pretende simular la realidad, para la práctica controlada de la escalada, colocando sobre esta estructura ya sea fija o móvil, toda una serie de elementos como presas, agarres, chapas, anclajes, reuniones, etc., que nos permiten progresar por ella, tanto horizontal como verticalmente, de una forma segura.

En relación al diseño, ésta es la parte en la que más tiempo y atención se ha prestado como bien indican Palacios y Bullido (2009), considerándola la parte más importante de todo el proyecto, ya que gracias a un buen trabajo de planificación se evitarán futuras modificaciones con todos los inconvenientes que esto pueda suponer.

Partiendo de la idea de la página Web de "Moonboard", ésta se basa en la construcción de un rocódromo fijo con una inclinación de $40^{\circ}$ sobre el que hay que

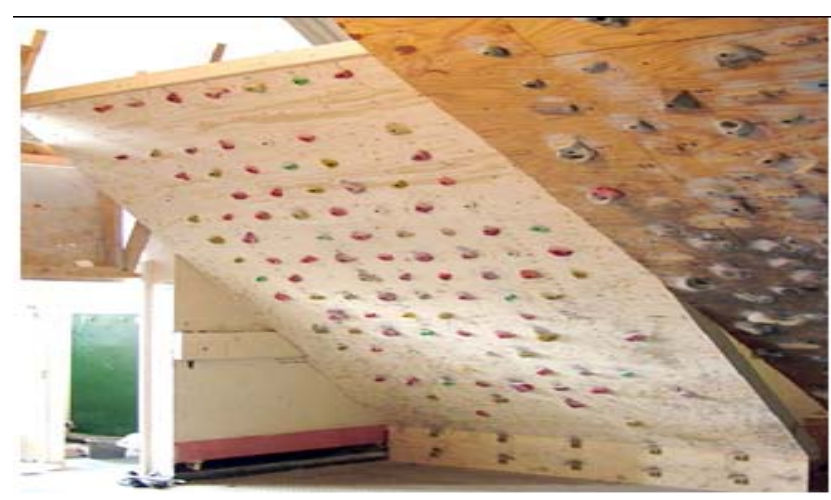

Figura 1. Imagen tomada de www.moonclimbing.com instalar unas presas diseñadas y suministradas por ellos mismos, para realizar una serie de movimientos según la dificultad o nivel del escalador. (Figura 1).

Pues bien, para el diseño del rocódromo se seguirán las siguientes fases:

1. Fase de Planificación

2. Fase de Elaboración

3. Fase de Ejecución

Fase 1.- Planificación: Se elaboraron unos planos con las medidas iniciales y se comprobó la idoneidad del espacio y materiales de instalación disponibles (paredes) para llevar a cabo la construcción del rocódromo y se estableció el ancho del plafón.

Para la construcción del modelo que presentamos a continuación está limitado por la anchura disponible. En el diseño original se estableció una anchura de $244 \mathrm{~cm}$ pero se amplió hasta los $315 \mathrm{~cm}$ para el aprovechar al máximo la pared de la que se disponía (Figura 2).

Fase 2.- Elaboración: Una vez validado el diseño se procedió a la construcción de todo el armazón metálico. Este trabajo se dejó en manos de personal cualificado llevándolo a cabo en una carpintería metálica (Figura 3).

Éste armazón está hecho a partir de perfil cuadrado de $35 \mathrm{~mm}$ por $35 \mathrm{~mm}$, con un bastidor de $37 \mathrm{~cm}$ de alto por 315 de ancho. En la parte baja, éste quedará fijado a la pared por medio de 12 parabolts. Sobre él están soldadas cinco bisagras y sobre estas, otro bastidor de 366cm de alto por 315 de ancho, que basculará sobre el bastidor inferior para obtener la inclinación deseada.

En la parte posterior se soldaron 8 anillas de acero para la sujeción del plafón superior a la pared, que se dividen según su función en 6 anillas de sujeción y de inclinación del nivel, y otras dos para el mecanismo de elevación y abatimiento del rocódromo. 


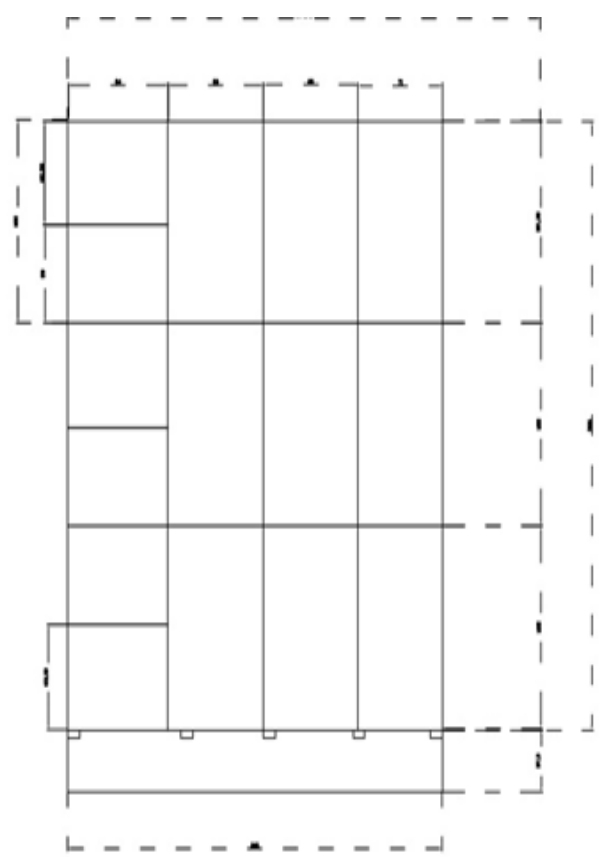

Figura 2. Plano inicial.

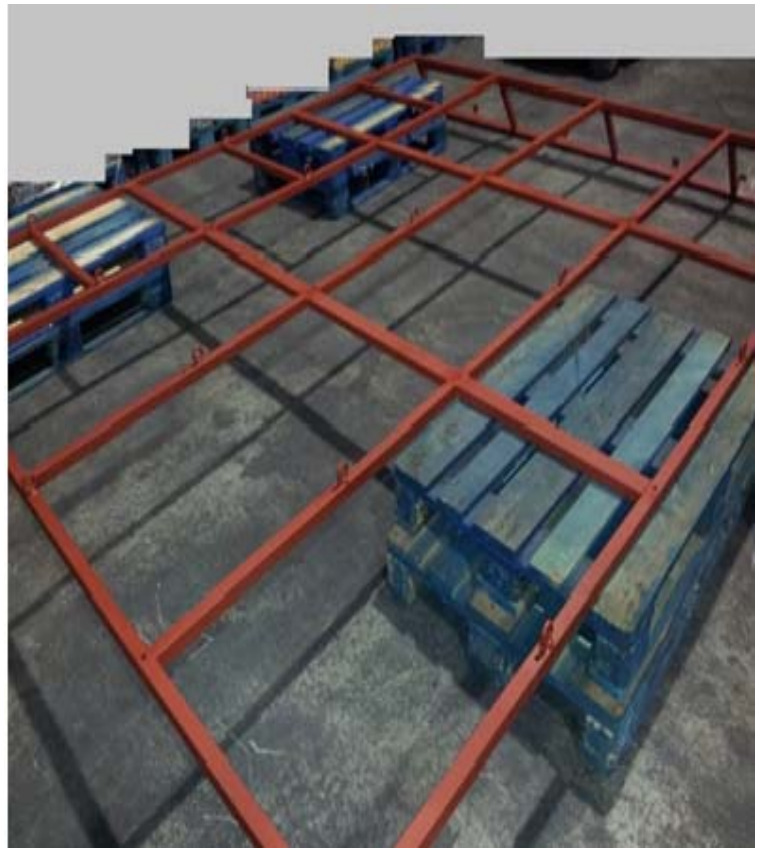

Figura 3. Armazón metálico

\section{Consideraciones a tener en cuenta}

Para establecer la separación entre tramo y tramo del armazón metálico (tanto los tramos horizontales como verticales) se tuvo en cuenta el futuro emplazamiento de los agujeros para las presas, de forma que no coincidiera ninguno de éstos con el lugar donde se fijarán las presas.

Se utilizaron tableros de densidad media (DM) de $122 \mathrm{~cm}$ por $244 \mathrm{~cm}$ y $19 \mathrm{~mm}$ de grosor, (Figura 4). Estos se fijarán al armazón metálico (con tornillos de cabeza de carro en el bastidor superior y con tornillos avellanados con cabeza de allen en el inferior) que previamente se habrán soldado las tuercas al armazón inferior por la parte posterior que quedará pegada a la pared. Una vez anclado a la pared no se podrá acceder a esta parte para sujetar las tuercas y apretar los tornillos.

Para establecer la distancia de separación entre presa y presa se fijaron los $20 \mathrm{~cm}$ que se indicaba en el diseño inicial, pero en lugar de fijar la primera presa a esa distancia del borde, se optó por hacerlo a $5 \mathrm{~cm}$ de este, distancia suficiente para librar el primer tramo vertical del armazón metálico (Figura 5) de la parte posterior que le dará consistencia a todo el conjunto una vez fijados todos los tornillos. De esta forma se aprovecha al máximo la superficie de la que se dispone.

Cada agujero lleva atornillado por la parte de detrás de los tableros una tuerca autoclavante de forma que nos resulte más cómodo colocar las presas, así como cambiar la disposición de las mismas. De esta forma evitaremos caer en la monotonía de realizar siempre los mismos ejercicios, modificando la ubicación o sustituyendo por nuevas una vez rotas o deterioradas las viejas.

Fase 3.- Ejecución: Tras la elaboración de las partes fundamentales del rocódromo, se pasó por último a la ejecución: el anclaje a la pared.

En el modelo que presentamos, para sujetar el plafón a la pared se instalaron 6 parabolt de $10 \mathrm{~mm}$ de diámetro por $120 \mathrm{~mm}$ de profundidad $(10 \mathrm{~mm} \varnothing \mathrm{X} 120 \mathrm{~mm})$ siendo el anclaje de expansión más fiable desde la escuela española espeleología y sistemas de fijación "Fischer" que para este tipo de superficie que garantizan una resistencia a la extracción de más de $2500 \mathrm{~kg}$.

Más otro parabolt a 1.5m por encima del borde superior para instalar el sistema de abatimiento y elevación del plafón.

Estos parabolt estarán rematados por una anilla de elevación del mismo diámetro que el parabolt. 
Para unir el armazón metálico con los parabolt fijados en la pared, se utilizó cable de acero trenzado de $8 \mathrm{~mm}$ y para que todos los puntos soportaran la misma carga se instalaron unos tensores, de forma que se podía ajustar la tensión de cada uno de ellos hasta dejar todo el peso del conjunto repartido equitativamente.

Todo este sistema descrito limitará la inclinación del conjunto, que en este caso la hemos fijado en torno a unos $40^{\circ}$ de inclinación máxima. (Figura 6)

Por ultimo cabría destacar que la mayor parte del peso de rocódromo está derivada al suelo ya que la estructura metálica reposa sobre el mismo y por tanto los anclajes solo soportan un porcentaje del peso total del rocódromo.

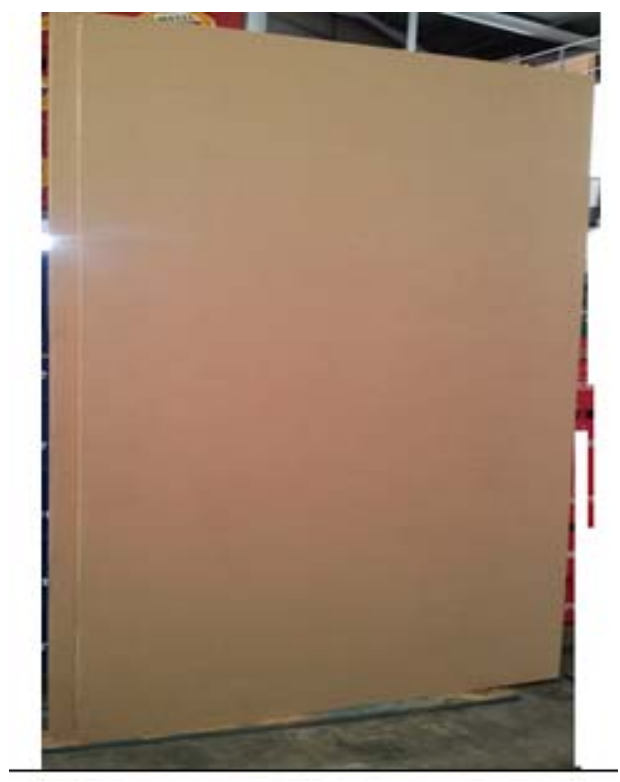

Figura 4. Tableros DM 122mm x 19mm

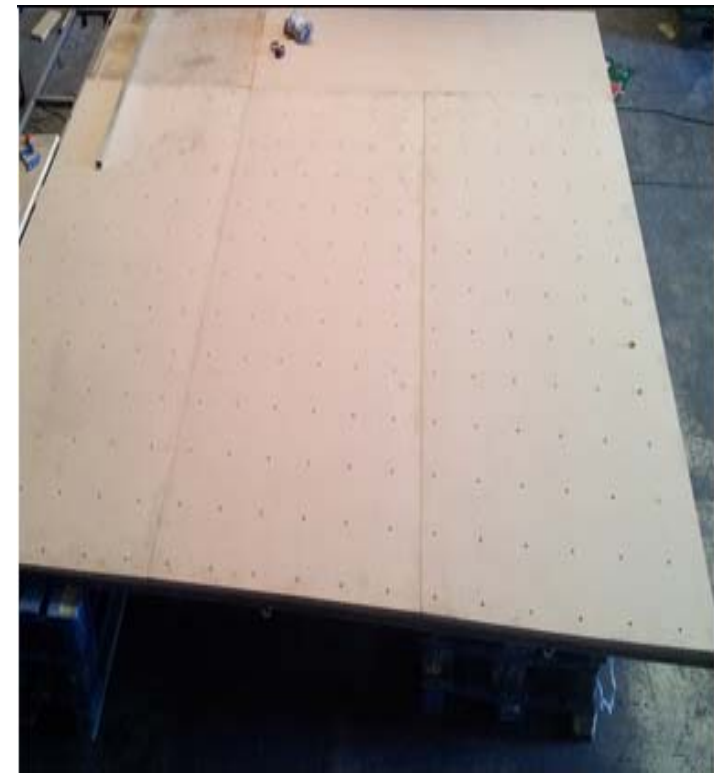

Figura 5. Detalle distancia entre agujeros

\section{Sistema de abatimiento y elevación del rocódromo}

Desde el parabolt descrito con anterioridad que está situado a $1.5 \mathrm{~m}$ por encima del borde superior del rocódromo se instaló un sistema de poleas dobles a la anilla central superior del armazón metálico que permite reducir la fuerza a realizar hasta $1 / 4$ del peso total del rocódromo. (Figura 7).

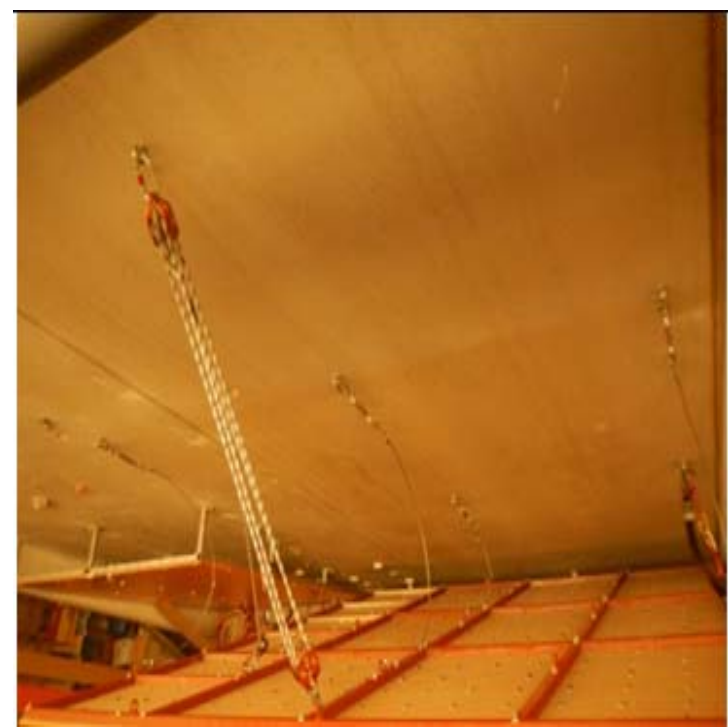

Figura 6. Detalle colocación de parabolt y anillas de sujeción.

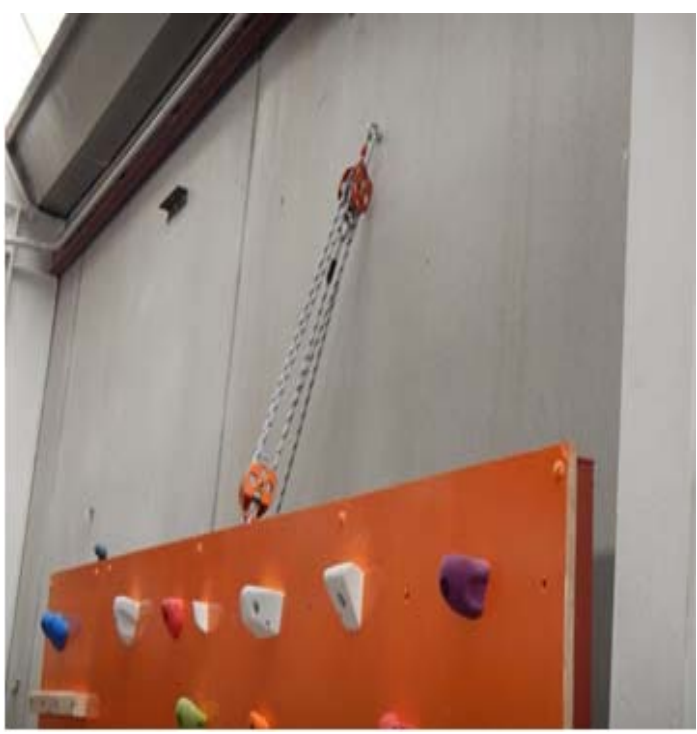

Figura 7. Detalle del sistema de poleas. 


\section{3.- CONCLUSIONES}

Como conclusiones podemos considerar que hemos intentado exponer una forma de construcción de un rocódromo de forma rápida y económica. Hemos intentado basarnos en la realidad de los centros educativos de nuestro país, y de la ausencia de materiales referentes a las actividades en el medio natural. También recordar que estas instalaciones se pueden construir junto con los ayuntamientos y así que pueda ser utilizado por el resto de la Comunidad Educativa en horario extraescolar, fomentando así también en nuestros alumnado el placer por estas actividades y que formen parte de su ocio y tiempo libre. El resultado final se puede observar en la Figura 8.

Además de todo ello, las ventajas de este rocódromo con respecto a otros publicados en otros trabajos, serían:

- Puede ofrecer diferentes niveles de dificultad según el grado de inclinación.

- Ofrece más versatilidad.

- Se adapta mejor a los diferentes ritmos del alum-

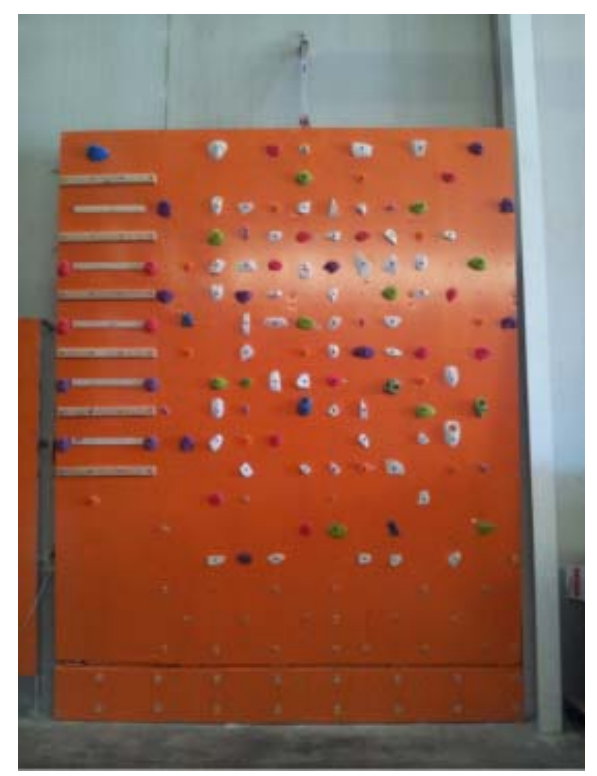

Figura 8. Vista frontal del rocódromo nado

- Aporta mayor creatividad y dinamismo a la hora de realizar ejercicios sobre él.

- Posibilidad de cambiar la ubicación de las presas fácilmente restando monotonía sobre otros que las tienen fijas.

\section{4.- REFERENCIAS}

Aguado, A. M. (2001). Actividades Físicas en el Medio Natural en la Educación Física Escolar. Cuadernos Técnicos $n^{\circ}$ 4. Palencia: Patronato Municipal de Deportes.

Baena Extremera, A. y Granero Gallegos, A. (2009). Deportes de Aventura Indoor: la espeleología en los institutos de Educación Secundaria. Tándem, Didáctica de la Educación Física, 30, 47-60.

Baena, A. y Calvo, J. F. (2008). Elaboración y construcción de materiales para el bloque de contenidos de Actividad Física en el Medio Natural: el rocódromo de escalada. Espiral. Cuadernos del Profesorado, 1(1), 1-8.

Beas, M. y Blanes, M. (2010). Posibilidades pedagógicas de la escalada en rocódromo. Espiral. Cuadernos del Profesorado, 3(5), 59-72.

Briongos, F. y Pérez O. (2008). Una estructura para escalar en la escuela. Wanceulen e.f. digital, 4, 163-173

Casero, O. (2007). Creación de un rocódromo interdisciplinar. Actas del VII Congreso Internacional sobre la Enseñanza de la Educación Física y el Deporte Escolar Pontevedra. Recuperado de: http://www.altorendimiento.com/congresos/instalaciones-y-equipamiento/452-creacion-de-unrocodromo-interdisciplinar

Palacios, J. M. y Bullido, E. (2009). Móntatelo en casa rocódromos personales, instalación y entrenamiento. Madrid: Desnivel.

Real decreto 1631/2006, de 29 de diciembre, por el que se establecen las enseñanzas mínimas correspondientes a la Educación Secundaria Obligatoria (B.O.E. $\mathrm{n}^{\circ} 5$ de 5 de enero de 2007). 